\title{
Standardization in Cyber-Physical Systems: The ARUM Case
}

\author{
Paulo Leitão ${ }^{1,2}$, José Barbosa ${ }^{1}$, Maria-Eleftheria Ch. Papadopoulou ${ }^{3}$, Iakovos S. Venieris ${ }^{3}$ \\ ${ }^{1}$ Polytechnic Institute of Bragança, Campus Sta Apolónia, Apartado 1134, 5301-857 Bragança, Portugal \\ \{pleitao,jbarbosa\}@ipb.pt \\ ${ }^{2}$ LIACC - Artificial Intelligence and Computer Science Laboratory, Rua Dr. Roberto Frias, 4200- 465 Porto, Portugal \\ ${ }^{3}$ School of Electrical and Computer Engineering, National Technical University of Athens, Athens, Greece \\ marelpap@icbnet.ece.ntua.gr, venieris@cs.ntua.gr
}

\begin{abstract}
Cyber-physical systems concept supports the realization of the Industrie 4.0 vision towards the computerization of traditional industries, aiming to achieve intelligent and reconfigurable factories. Standardization assumes a critical role in the industrial adoption of cyberphysical systems, namely in the integration of legacy systems as well as the smooth migration from existing running systems to the new ones. This paper analyses some existing standards in related fields and presents identified limitations and efforts for a wider acceptance of such systems by industry. A special attention is devoted to the efforts to develop a standardcompliant service-oriented multi-agent system solution within the ARUM project.
\end{abstract}

\section{INTRODUCTION}

Markets are imposing strong requirements for industrial companies running their businesses, namely demanding highly customized products exhibiting high quality and reduced prices. Future automation systems will need to incorporate new methodologies and technologies allowing to be more adaptable to react to these changing requirements, as well as to new product variants/models. Challenges are basically related on implementing more complex systems, able to collaborate with other systems, exhibiting reconfigurability and responsiveness in a quite natural manner.

This vision is sustained, e.g., by Industrie 4.0 that promotes the computerization of traditional industries to achieve intelligent factories characterized by adaptability, efficiency, functionality, reliability, safety, and usability, and supporting the integration of customers and business partners [1]. This vision considers distributed intelligence and self-* methods, namely self-adaptation, self-configuration and self-diagnosis.

The emerging concepts of Internet of Things and CyberPhysical Systems (CPS) promise a breakthrough in achieving interoperability, flexibility and reconfigurability of devices and systems, being capable of seamless reconfiguration and more efficient in productivity and energy consumption.

Cyber-physical systems can be defined as the combination of mechatronics and IT to control physical processes and systems, designed as a network of interacting software and hardware devices and systems, each one with a higher level of autonomous decision making. CPS focuses on intelligent, dynamic and self-* large-scale systems, covering the interand intra-enterprise integration and running in ubiquitous environments. Examples are manufacturing and process plants, electrical power grids and pipelines, logistics and transportation. Particularly in manufacturing, a moving from the existing hierarchical control structures based on the ISA 95 automation pyramid towards more decentralized and reconfigurable structures based on the CPS principles is being noticed, as illustrated in Fig. 1.

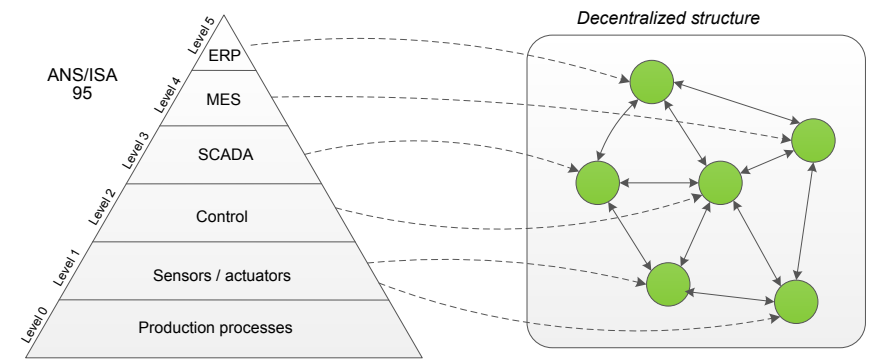

Fig. 1. Moving from ISA 95 towards cyber-physical production systems

The complete adoption of such concepts and technologies, where the current "nice-to-have" should lead to the "musthave" in the future, requires the convincement of industry stakeholders, the availability of sound software engineering methodologies, and the achievement of industrial requirements, particularly in terms of resilience, security and privacy. Smooth migration, another important challenge in the development of such CPS solutions, "should be implemented gradually through the digital upgrading of existing facilities, which means that the concept can be realized by upgrading existing sites and not just in newly built facilities", as referred by Steve Sands from Festo [2].

Standardization assumes a critical role in the industrial adoption of CPS solutions since standards may affect the development, installation and commissioning of industrial applications. For example, standardization can support the deployment of CPS, and particularly the smooth migration, by easily interfacing with existing legacy systems, plug-andplay systems, devices and algorithms, and adapting their behaviour and relationships on-the-fly.

The objective of this paper is to understand the importance of standardization in the development of CPS fulfilling the 
industrial requirements and permitting a smooth migration from the traditional control systems to the new Industrie 4.0 vision. Some existing standards are analysed, identifying their limitations and the current standardization efforts and initiatives promoted by $\mathrm{R} \& \mathrm{D}$ projects developing CPS for a wider acceptance of such systems by industry. A special attention is devoted to the efforts to develop a standardcompliant service-oriented multi-agent systems solution within the ARUM project.

The rest of the paper is organized as follows: Section II overviews the existing standards in this field and points out limitations and missing standardization issues to fulfil the industrial requirements. Section III presents some standardization efforts and initiatives related to the implementation of CPS applications and Section IV describes the efforts developed within the ARUM project to achieve standard-compliant solutions. At last, Section V rounds up the paper with the conclusions.

\section{EXISTING STANDARDS SUPPORTING CPS SOLUTIONS}

The development of CPS solutions involves the use of heterogeneous hardware and software technologies, organized as a network of distributed control nodes, some of them interconnected with legacy systems and hardware devices. The implementation of such systems being compliant with standards is crucial for their industrial adoption.

Standards define a set of understandable and unambiguous rules to regulate products, processes or services, ensuring quality, safety, security and interoperability [3]. As a result, standards are crucial to ensure that products and systems work as expected, are of desired quality and compatible with other equipment. Standards are established by standardization bodies, with a national or international scope, e.g., IEEE (Institute of Electrical and Electronics Engineers), IEC (International Electrotechnical Commission) and NIST (National Institute of Standards and Technology).

Several categories of standards have to be supported by CPS to facilitate the industrial adoption, mainly: distributed intelligent control technologies, interoperability, industrial communication networks, security and interface with hardware devices (e.g., providing real-time control). Also, some standards dependent on the application domain should be considered

\section{A. Distributed Intelligence}

The distributed control strategies inherent in CPS solutions, can be implemented by using, e.g., Multi-Agent Systems (MAS) or Service-Oriented Architecture (SOA).

The Foundation for Intelligent Physical Agents (FIPA) [4] produced specifications for heterogeneous agent-oriented software engineering, being currently the only standard for the development of MAS systems. FIPA specifications are grouped in different categories [5]: applications, abstract architecture, agent communication, agent management and agent message transport. The FIPA Applications specifications refer to the application areas where FIPA agents can be deployed, representing ontology and service descriptions specifications for a particular domain. The FIPA Abstract Architecture specifications deal with abstract entities that are required to build agent services and an agent environment. The FIPA Agent Communication specifications deal with the Agent Communication Language (ACL), interaction protocols, speech act theory-based communicative acts and content language representations. In particular, it includes the FIPA Interaction Protocols specifications that define the pre-agreed protocols for ACL messages exchanged between agents. The FIPA Agent Management specifications deal with the control and management of agents within and across agent platforms, and specify a reference model that defines the basic structure of a MAS compliant with FIPA. At last, the FIPA Agent Message Transport specifications deal with the transport and representation of messages across different network transport protocols.

FIPA specifications miss several important industrial requirements, namely:

- Security and privacy.

- Hardware and legacy systems integration.

- Real-time interaction protocols for large-scale systems, ensuring scalability and latency.

- Light ACL protocol to support scalability in large-scale systems (note that the format protocol of FIPA-ACL is too heavy mainly due to the headers in the fields, being required the reduction of their size).

- Distributed yellow pages service and discovery mechanism to improve the system robustness.

Out of the FIPA organization, the Agent Unified Modelling Language (AUML) initiative extends the UML language to address the requirements of large-scale agent-based applications [6].

In the field of SOA, several standards can be identified. Commonly SOAs are based on web services (WS), using standard and open protocols to provide a communication platform between distributed and heterogeneous systems and applications. Most of the web service platforms are made of SOAP (Simple Object Access Protocol), WSDL (Web Services Description Language) and UDDI (Universal Description, Discovery and Integration), which use the combination of HTTP (Hypertext Transfer Protocol) and XML (Extensible Markup Language) as the basic foundation of WS (see Fig. 2).

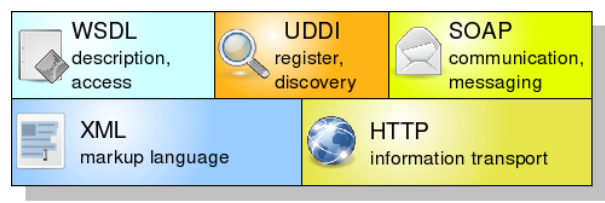

Fig. 2. Web service's building blocks

The capabilities of registration and discovery of services are achieved by the use of UDDI directory facilities and the service description can be performed by using WSDL description structures. The WSDL description service provides functionalities of how to call the service, the input 
parameters and expected outputs. SOAP defines the exchange of structured and typed information between peers. Among others, the SOAP standard defines the way the exchanged envelope is structured.

Reference models were also developed to guide the development of SOA systems. Particularly, the OASIS (Organization for the Advancement of Structured Information Standards) [7] reference architecture and more recently the open group SOA Reference Architecture [8] are defining guidelines and options for the architectural design and implementation of decision-making.

Recognizing the importance of combining agents with web services, FIPA set up the "Agents and Web Services Interoperability Working Group" aiming to define standards allowing agents to be able to interact with Web services seamlessly and vice versa.

\section{B. Interoperability}

In heterogeneous CPS systems, the interoperability and the understanding of the shared knowledge during cooperation processes is an important aspect. For this purpose, standards addressing interoperability, information exchange and interfaces to legacy systems must be considered.

A first issue is related to the knowledge representation shared by the distributed entities. A proper way to standardize the shared knowledge is to use ontologies, since they provide a common representation of the knowledge handled by the distributed agents. In this domain, several standards were established, mainly driven by World Wide Web Consortium (W3C) and OASIS. The existing standards are mostly based on XML, being RDF (Resource Description Framework) [9] and OWL (Web Ontology Language) [10] the best known ones. OWL has a rich set of modelling constructors, offering predefined templates, e.g., supporting the inclusion of restrictions in the concepts and predicates.

Aiming the real interoperability within CPS solutions, another important issue is the use of standard interfaces for the integration of legacy systems. Classical OPC (OLE for Process Control) uses the Microsoft's COM/DCOM communication technology while defining standards for data access (including alarms and events). The OPC-UA (Unified Architecture) aims to push forward the COM/DCOM limitations by using the state-of-the-art communication technology, such as web services, allowing to deal with security and platform independency issues. By doing so, OPC-UA is becoming a de facto standard for enabling the transparent, multi-vendor, interoperability, allowing the cross vertical communication among the ISA 95 layers.

\section{Industrial Communication}

Industrial communication plays a crucial role in the CPS world, once it is responsible for guaranteeing real-time, safe and secure data transmission at the shop-floor level. There are a plethora of standards and de facto standards, such as CAN (ISO 11898), EtherCAT (IEC 61158), ModBus (de facto) and Profibus (IEC 61158/IEC 61784). Ethernet Powerlink is a promising technology aiming to be the real-time industrial Ethernet, being currently in a standardization process.

The wireless communication is also being used as a fast way for technology upgrade in existing shop-floors or simply as the way to reduce communication costs, e.g., by avoiding the use of cables. In this domain, several technologies should be considered, namely Industrial WLAN (IWLAN), WirelessHART and Zigbee, which are based upon the IEEE 802.15.4 standard.

\section{Security and Privacy}

As CPS are built upon cyber and physical infrastructures, incorporating different strategies and architectures, such as MAS and SOA, they become vulnerable to a variety of threats and attacks due to the combination and interaction of the aforementioned components and technologies. Thus, the latest security practices and technologies should be considered to address different kinds of either external or internal threats, identify vulnerabilities and provide in general: a) physical security, preventing unauthorised personnel or even accidental intruders from having access to software and hardware devices, b) network security, protecting the communication channels against malicious activities or cyber-attacks taking different forms like worm, virus infiltrations or Denial of Service (DoS) attacks, and c) IT and data security, protecting information from actions of unauthorised users, to ensure confidentiality, integrity and availability of data and resources.

The adoption of security standards for secure communication, authentication and authorisation, as well as policy definition and enforcement is essential. As far as securing communication between system components is concerned, message encryption is a very common technique to ensure confidentiality of the information exchanged. W3C developed the XML-Encryption standard [11], which defines how to encrypt the contents of an XML element, hence it can be used to secure communication schemas that use XML messages, e.g., RDF. Moreover, the XML-Signature [12], standardised by $\mathrm{W} 3 \mathrm{C}$, can be applied to any digital content, e.g., SOAP messages, for the sake of non-repudiation and integrity of the exchanged messages.

With regard to authentication, the Security Assertion Markup Language (SAML) [13] is an XML-based, transport independent open standard produced by OASIS, which implements a request/response pattern for exchanging user authentication information between an Identity Provider and a Service Provider. In addition, Role-Based Access Control (RBAC) [14] was standardised by NIST as an authorisation mechanism to provide access to users by means of policy enforcement. With respect to policy specification and enforcement, OASIS developed a standard to complement SAML with a flexible authorisation system known as eXtensible Access Control Markup Language (XACML) [15], which is an attribute-based XML-based standard for defining access control policies and evaluating access requests according to the rules defined in policies. 


\section{E. Interfaces for Low-level Control}

The interconnection with hardware devices performing (real-time) control is mandatory in CPS. Since agents mainly address the high-level of abstraction, they are useful to provide autonomy and intelligence but miss real-time constraints. The development of standard-compliant solutions to achieve real-time control systems requires the creation of an agent layer to provide intelligence and adaptation, preserving the current automation low-level control to guarantee real-time responsiveness.

The low-level control can use state-of-the-art standard technologies implementing industrial control, namely IEC 61131 standard for Programmable Logic Controllers (PLC) [16] and IEC 61499 for distributed automation [17]. These two levels, as illustrated in Fig. 3, are interconnected by exchanging data through a PLC data table. The proprietary interface and the missing standard semantics in the exchanged information are the disadvantages of this solution.

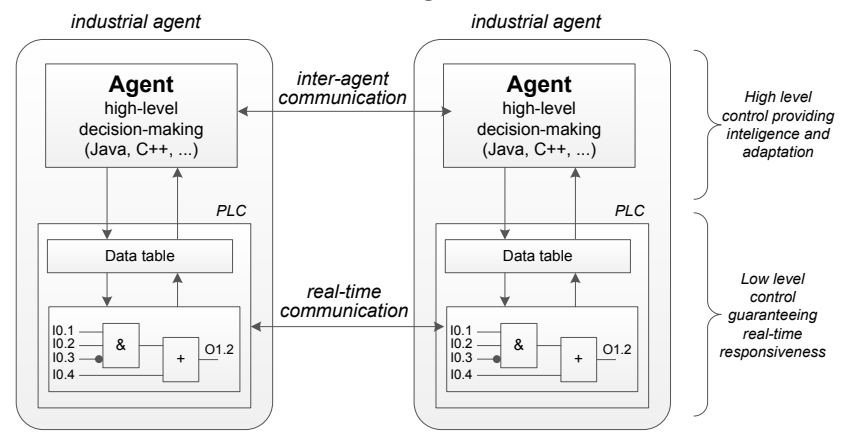

Fig. 3. Industrial agents using standard IEC 61131 interfaces

The deployment of industrial agents can also be envisioned by using the recent trend of open hardware platforms, e.g., Arduino, or the small computer boards, e.g., RaspberryPi.

\section{F. Application Domain-dependent Standards}

Besides the described standards that generally may influence the deployment of industrial CPS systems, other standards dependent on the automation application domain should be considered. For example, ANSI/ISA95 is a standard for the development of automated interfaces between enterprise and control systems, being applied in all industries, and in all sort of processes, such as batch, continuous and repetitive processes, and IEC 61850 is a standard for the design of electrical substation automation to be considered in smart grids field. ANSI/ISA 88 is a standard addressing batch process control, being a design philosophy for describing equipment and procedures, and not focused on software. ISO 15531 is a standard providing models for the representation of data that is captured from the manufacturing control level, and stored and handled at the manufacturing management level.

Product definition data is one important step in the product life-cycle management. As far as it is concerned, ISO 14306 [18] has defined a file format for capturing and repurposing of lightweight $3 \mathrm{D}$ product definition data. This binary file standard, also known as JT file, is used as the mean for product visualization, collaboration and $\mathrm{CAD}$ data exchange. Also important is the consideration of the open standard AutomationML (Automation Markup Language), which is a neutral data format based on XML for the storage and exchange of plant engineering information, to interconnect heterogeneous engineering tools from different fields, e.g., mechanical plant engineering, electrical design and PLC.

Similarly, special attention should be devoted to standards for user interfaces, especially those based on web technologies, HMI (Human-Machine Interface) and SCADA (Supervisory Control and Data Acquisition). Moreover, regarding HMI and SCADA standards, ISA101 HMI standard is about to be issued at the end of 2014, providing the appropriate guidance for designing, implementing and managing human-machine interfaces in manufacturing applications [19].

\section{STANDARDIZATION EFFORTS AND INITIATIVES}

Recently, and showing the importance of standardization to drive innovation and increase productivity by supporting the development of new technologies, there is a tendency to incorporate specific tasks dealing with standardization activities within the EU R\&D projects. These efforts are mainly related to push the development of standard-compliant approaches and technologies and also to interact with standardization bodies to articulate specific requirements emerged from the $R \& D$ activities performed during the projects. Examples of such projects in the field of CPS, and particularly in the MAS domain, are GRACE (inteGration of pRocess and quAlity Control using multi-agEnt technology), IDEAS (Instantly Deployable Evolvable Assembly Systems), ARUM (Adaptive Production Management) and PRIME (Plug and produce intelligent multi-agent environment based on standard technology).

For example, the GRACE project [20] developed and installed a MAS monitoring and control computational system in a factory plant producing laundry washing machines, respecting the standards. Namely, the agent-based system was developed using JADE (Java Agent Development Framework) [21], which is FIPA compliant, the exchange of messages among the agents used FIPA-ACL, and the designed ontology was expressed in the OWL language. Also, the low-level control running IEC61131 programs on PLCs were preserved to ensure the real-time responsiveness and the easy and transparent access with the agents.

In United States, industry and Government have posed CPS at the center of the engineering research agenda since 2007, constituting an opportunity to achieve innovation and competitiveness by implementing smart systems that exhibit complex behaviour patterns due to embedded software components. NIST is playing an important role to promote the deployment and standardization of CPS, developing efforts with several federal organizations and commercial companies, such as IBM, Intel, GE and Boeing. To be more 
specific, the NIST SmartAmerica Challenge pretends to be a playground where several teams are demonstrating how CPS can improve manufacturing, health care, emergency response, transportation and others while fostering jobs and economic growth.

Other initiatives being developed by several working groups and technical committees near standardization bodies can be reported. Inside the IEEE Technical Committee on Industrial Agents, a working group is studying and compiling a set of recommendation practices for applying agent technology in industrial environments, which posteriorly can derive opportunities for extending existing standards or generate new ones. Particularly, the collaboration with FIPA was established to identify new specifications addressing industrial requirements. The VDI/VDE GMA Technical Committee 5.15 on Multi-Agent Systems is also working in standardization issues of MAS, reflected in a set of guidelines compiled in [22].

As far as security in CPS is concerned, due to the complexity and heterogeneity of the system, the use of current security frameworks and privacy-preserving tools need to be reconsidered as new security concerns are introduced. It is essential that an overall security system architecture is designed, taking into account both cyber and physical characteristics of the infrastructure, according to the CPS application domain [23]. To be more specific, due to the diversity of the entities that are involved in CPS, responsible to collect, generate and modify data, stronger cryptographic tools should be used and access control mechanisms should be scalable, attribute-based as well as context-aware, in order to ensure data authenticity and system trustworthiness, especially in large-scale critical infrastructures [24].

\section{STANDARDIZATION IN THE ARUM PROJECT}

As described before, standardization activities are being included in the work programme structure of $\mathrm{R} \& \mathrm{D}$ projects as a key to achieve industrial-driven goals. This section details how standardization is being considered within the ARUM project during the development of the proposed platform.

\section{A. Overview of the ARUM System}

The ARUM (Adaptive Production Management) project [25] addresses the development of novel Information Communication Technology (ICT) solutions to handle new challenges in ramp-up production of complex and highly customized products, such as aircraft and shipbuilding industries. These CPS focus on the development of mitigation strategies to respond faster to unexpected events by implementing systems and tools for the decision support in planning and operation [26]. For this purpose, the ARUM platform uses an intelligent Enterprise Service Bus (ESB) that enriches the traditional ESB with a plethora of advanced modules, such as Ontology service, Data Transformation Service, Sniffer, Node Management and Life-Cycle Management. The iESB provides a common infrastructure for the integration of heterogeneous agent-based planning and scheduling tools and legacy systems, as illustrated in Fig. 4.

Under the scope of the project, several planning and scheduling agent-based tools were developed, such as the Operational Scheduler (OS) and the Strategic planner (SP). The plugability of the agent-based tools is facilitated by the exposition of their functionalities as services and by the use of ontology services for the representation of the shared knowledge, improving the interoperability in such distributed and heterogeneous systems.

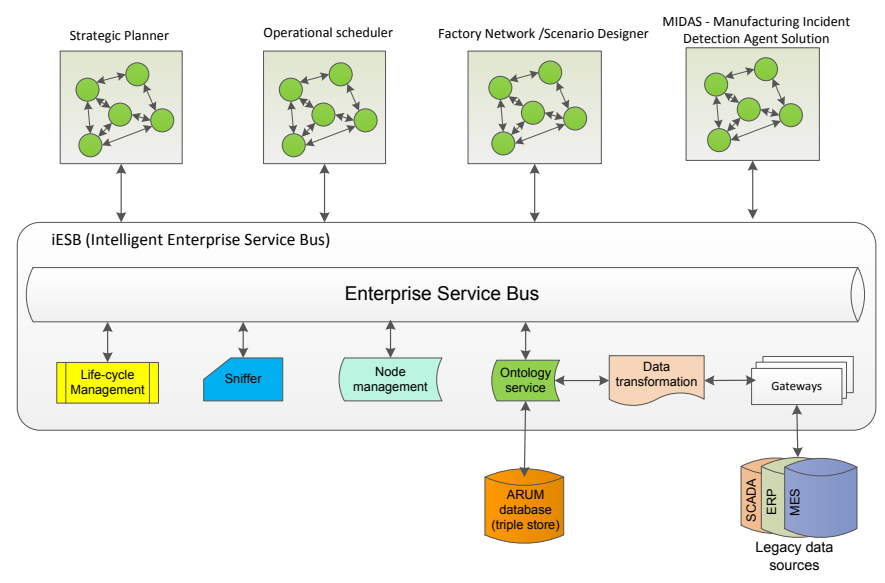

Fig. 4. ARUM platform architecture

\section{B. Standard-compliance in the ARUM Solution}

Core components of the ARUM platform, like the OS and the SP, are implemented using agent technologies under the FIPA specifications. Interoperation and negotiation patterns between agents, as well as between multiple multi-agent schedulers in a holonic perspective, are required for the real time scheduling in industrial applications and therefore the opportunity of establishing related standards. Moreover, due to the fact that the aforementioned technology is used, the format of messages exchanged between the components within the ARUM platform should be also conformant with the FIPA Interaction Protocols. In such way, ARUM is promoting the integration of the FIPA interaction protocols with the service-orientation principles, enabling a proper communication mechanism for the system dynamics, e.g., when several distributed schedulers are cooperating. In such way, the OSs can interact among each other using the already known negotiation protocols developed under FIPA.

As ARUM follows an ontology-driven approach to achieve its operational goals, apart from complying with the international standards, e.g., design using OWL, it has the potential to contribute to the evolution of the latter and the use of the RDF format guarantees the proper information exchange between tools or data access to the legacy systems. Ontological information models for the semantic description of the data regarding the discrete production processes, the actual and alternative scenes of the production line, the sensor data collected during the assembly processes along with the associated events and access control policies based on attributes for authorisation decisions, have been developed as 
well as the appropriate tools for their management. Additionally, a REST based API accessible only over HTTPS is used as the way for GUIs to have secure access to the data to be displayed.

Interoperation and compliance with legacy systems used in manufacturing processes, such as Enterprise Resource Planning (ERP), Supervisory Control and Data Acquisition (SCADA) and Customer Relationship Management (CRM), is of great importance. Having this in mind, it is essential that ARUM comply with standards regarding industrial automation and business interoperability and communication. Since the development of the platform and tools is grounded on concepts like service-orientation, this creates potentials for contributing to standardisation bodies including, for example, W3C initiatives with semantic enhancement of service descriptions. Additionally, ARUM is pushing the state-of-theart by promoting a combination of data transformation mechanisms from legacy systems with the ARUM ontology, allowing a cross-access of data within all tools in a seamless way through the ontology service.

As far as industrial automation is concerned, according to ANSI/ISA95, ARUM addresses interoperability at Level 3, where the agents' network is positioned, as well as at Level 4, supporting the integration with legacy systems. Compliance with standards related to the aforementioned levels and industrial operations in general is required, including IEC/ISO 62264 for enterprise control systems integration, ISO/IEC 15288 regarding lifecycle management and ISO 15531 for manufacturing management data exchange.

\section{CONCLUSIONS}

The Industrie 4.0 initiative towards a computerization of traditional industries aiming to achieve intelligent factories is demanding the consideration of new paradigms, such as Internet of Things and CPS. In particular, the implementation of CPS considers several hardware and software technologies, which are integrated to support distributed, modular, robust and re-configurable solutions.

Standardization assumes a critical role in the industrial adoption of the concepts and technologies involved in CPS, as well as the smooth migration from existing running systems to the new ones. In fact, the understanding and adoption of the plethora of standards associated to the CPS technologies supports the deployment of standards-compliant CPS solutions.

This paper analysed the existing standards in the field, identified limitations to face the current requirements imposed by CPS, and described on-going standardization efforts for a wider acceptance of such systems by industry. A special attention was devoted to the efforts for the development of a standard-compliant service-oriented multiagent systems solution within the ARUM project.

\section{ACKNOWLEDGMENT}

The research leading to these results has received funding from the European Union Seventh Framework Programme FP7 ARUM project, under grant agreement $n^{\circ} 314056$.

\section{REFERENCES}

[1] Böhler, T., "Industrie 4.0 - Smarte Produkte und Fabriken revolutionieren die Industrie", Produktion Magazin, 10 Mai 2012 (accessed on 3/4/14)

[2] Sands, S., "Industry 4.0, What's in a Name?", Industrial Technology, February, 2014.

[3] Seixas, I., Leitão, P., "Standards Compliance in Industrial Agents Applications", Proceedings of the 39th Annual Conference of the IEEE Industrial Electronics Society (IECON'13), pp. 7438-7443, 2013.

[4] FIPA, Foundation for Intelligent Physical Agents, www.fipa.org/ (available on 17/10/2014).

[5] Mařík, V., Pěchouček, M., Vrba, P., and Hrdonka, V., "FIPA Standards and Holonic Manufacturing", Deen, S. (ed.), Agent-based Manufacturing: Advances in the Holonic Approach, Springer Verlag, pp. 89-121, 2003.

[6] Bauer, B., Odell, J., "UML 2.0 and Agents: How to Build Agent-Based Systems with the New UML Standard", Journal of Engineering Applications of Artificial Intelligence, 18(2), 2005.

[7] OASIS, "Reference Architecture Foundation for Service Oriented Architecture Version 1.0", OASIS Service Oriented Architecture Reference Model Technical Committee, 4 December 2012.

[8] SOA Reference Architecture, The Open Group, www2.opengroup.org/ogsys/catalog/C119 (accessed on 24/5/2014)

[9] World Wide Web Consortium (W3C), "Resource Description Framework", 2004 (available at www.w3.org/RDF/ on 23/3/2013).

[10] World Wide Web Consortium (W3C), "OWL Web Ontology Language Reference", W3C Recommendation, 2004 (available at www.w3.org/TR/owl-ref/ on 23/3/2013).

[11] World Wide Web Consortium (W3C), "XML Encryption Syntax and Processing", W3C Recommendation, 2002 (available at www.w3.org/TR/2002/REC-xmlenc-core-20021210/ on 15/5/2014).

[12] World Wide Web Consortium (W3C), "XML Signature Syntax and Processing (2nd Edition)", W3C Recommendation, 2008 (available at www.w3.org/TR/xmldsig-core/ on 15/5/2014).

[13] OASIS, "OASIS Security Services (SAML) TC" (available at www.oasis-open.org/committees/tc home.php?wg abbrev=security on $15 / 5 / 2014)$.

[14] NIST, "The NIST Model for Role-Based Access Control: Towards A Unified Standard", (available at csrc.nist.gov/rbac/sandhu-ferraiolokuhn-00.pdf on 15/5/2014).

[15] OASIS, "OASIS eXtensible Access Control Markup Language (XACML) TC", (available at www.oasisopen.org/committees/tc_home.php?wg_abbrev=xacml on 15/5/2014).

[16] International Electrotechnical Commission (IEC), "IEC 61131: Programmable Controllers - Part 3: Programming Languages", Standard, 3rd Edition, 2012.

[17] International Electrotechnical Commission (IEC), "IEC 61499: Function blocks - Part 1-4", Standard, 2nd Edition, 2012.

[18] ISO 14306, "Industrial automation systems and integration - JT file format specification for 3D visualization", 2012 (available at www.iso.org/iso/home/store/catalogue_ics/catalogue_detail_ics.htm?cs number $=60572$ on $24 / 5 / 2014$ ).

[19] ISA101, Human-Machine Interfaces, www.isa.org/isa101/ (accessed on 22/10/2014)

[20] Rodrigues, N. Pereira, A., Leitão, P., "Adaptive Multi-agent System for a Washing Machine Production Line", Industrial Applications of Holonics and Multi-agent Systems, LNCS, vol. 8062, Springer, pp. 212-223, 2013

[21] Bellifemine, F., Caire, G., Greenwood, D., "Developing Multi-Agent Systems with JADE", Wiley, 2007

[22] VDI/VDE 2653, "Multi-agent systems in industrial automation", June 2010.

[23] Cardenas, A. et al., "Challenges for Securing Cyber Physical Systems", Workshop on Future Directions in Cyber-physical Systems Security, DHS, July 18, 2009.

[24] Michael, K., Book Review: Handbook on Securing Cyber-Physical Critical Infrastructure: Foundations and Challenges (written by Sajal K. Das, Krishna Kant, Nan Zhang), 2012. 
[25] ARUM - Adaptive Production Management, www.arum-project.eu/ (accessed on 17/10/2014).

[26] Marín, C., Mönch, L., Leitão, P., Vrba, P., Kazanskaia, D., Chepegin, V., Liu, L., Mehandjiev, N., "A Conceptual Architecture Based on Intelligent Services for Manufacturing Support Systems", Proc. of the IEEE International Conf. on Systems, Man, and Cybernetics (SMC'13), pp. 4749-4754, 2013. 